\title{
Magnetic Oscillations in Radiative Stars
}

\author{
E. J. Zita
}

The Evergreen State College, Olympia, WA, 98505 USA

\begin{abstract}
What drives acoustic modes in magnetic, radiative stars, such as the rapidly oscillating Ap (roAp) stars? We propose that oscillations in the shape of the magnetic field can drive magnetosonic waves, which are observed as $\mathrm{p}$ modes. The force-free atmosphere minimizes magnetic energy. If the field is strong enough, the magnetic configuration can oscillate about its equilibrium state. Initial calculations showed that the wavenumbers, frequencies, and available energy are consistent with observations of roAp stars (Zita 1997). We present the results of new calculations and proposed tests of our model. We also discuss the resultant $\langle v \times b\rangle$ dynamo, which may shed light on observations in B stars.
\end{abstract}

\section{Overview}

The source of $p$ modes in roAp stars is an unanswered question. Several hypotheses have been proposed in recent years. The opacity mechanism familiar in $\delta$ Scuti stars is damped by radiation in roAp ionization zones (Dziembowski \& Goode 1996). An attempt to use the $\kappa$ mechanism by imposing a steep edge temperature rise leads to unobserved long-period modes (Gautschy, Saio, \& Harzenmoser 1998), which are then theoretically suppressed by invocation of selective He draining. However, important new calculations (Matthews, Kurtz, \& Martinez 1999) suggest that roAp stars may be cooler than previously believed, not warmer. Interesting new work builds on Shibahashi's (1983) magnetoconvection model to describe p-mode selection in the weakly magnetic (dipole) dense body of roAp stars, where oscillations have low amplitude and high energy (Balmforth et al. 2000). Our model builds on Berger's (1985) force-free field solutions to describe p-mode driving in the strongly magnetic (multipole) atmosphere of roAp stars, where oscillations have high amplitude and low energy.

\section{Magnetic Oscillations in A Stars}

In the dynamic edge of roAp stars, where $\mathrm{mHz} p$ modes are observed (Kurtz 1990), the force-free magnetic field has a twisted multipole shape (Zita 1999). Small perturbations to this equilibrium configuration can increase the twist of the field in one hemisphere and decrease the twist in the other, conserving total helicity. These longitudinal oscillations in the mean magnetic field $(b)$ induce, by Faraday's law, a perpendicular oscillating electric field $E$. The familiar $E \times B$ drift drives compressional velocity fluctuations $(v)$. (At $10^{-5}$ the mean field 
strength, $b$ is below detection limits, for velocity fluctuations of $200 \mathrm{~m} / \mathrm{s}$.) These are simply magnetosonic waves in a spherical shell. Hydrostatic equilibrium calculations yield $\mathrm{mHz}$ magnetosonic frequencies for stars with $1.5-2.5 \mathrm{M}_{\odot}$ and field strengths between 1-2 kG. We find that frequencies generally increase with mass and decrease with field strength. Our prediction that multipole magnetic fields drive multipole $(\ell \geq 1) \mathrm{p}$ modes can be tested by measuring the distribution of surface metal spots (Kuschnig 1998) and by comparing equivalent widths of hydrogen and metal absorption lines (Viskum et al. 1998) Low-amplitude oscillations in the underlying dipole region may perturb the star's multipole magnetic surface, driving these trapped magnetosonic waves. Thus, the p modes are resonant with magnetic oscillations about a magnetic energy well.

\section{Turbulent Dynamo and B Stars}

Nonlinear coupling between fluid compressions $(v)$ and magnetic oscillations $(b)$ can result in a mean electric field $E_{\text {dynamo, }}$, which can sustain the mean magnetic field (Zita 1999). This $\langle v \times b\rangle$ dynamo mechanism may be more efficient in radiative stars than the usual $\alpha-\omega$ dynamo, if convection is lacking. New observations suggest magnetic fields in B stars (Smith \& Robinson 1999). Nonmagnetosonic oscillations may also contribute to this dynamo in very hot stars. The strength of the dynamo depends crucially on the phasing of the $v$ and $b$ oscillations. Further investigation requires nonlinear computational modeling.

Acknowledgments. This work has been supported by the National Science Foundation grant AST98-06188. We gratefully acknowledge helpful discussions with M. Berger, N. Evans, J. Fabregat, S. Frandsen, D. Gough, H. Shibahashi, and W. Weiss.

\section{References}

Balmforth, N., Cunha, M. S., Dolez, N., et al. 1999, in these proceedings, p. 453

Berger, M. A. 1985, ApJS, 59, 433

Dziembowski, W. A. \& Goode, P. R. 1996, ApJ, 458, 338

Gautschy, A., Saio, H., \& Harzenmoser, H. 1998, MNRAS, 301, 31

Kurtz, D. W. 1990, ARA\&A, 28, 606

Kuschnig, R. 1998, in Contrib. Astron. Obs. Skalnaté Pleso, 27, 418

Matthews, J. M., Kurtz, D. W., \& Martinez, P. 1999, ApJ, 511, 422

Shibahashi, H. 1983, ApJ, 275, L5

Smith, M. \& Robinson, R. 1999, ApJ, 517, 866

Viskum, M., Kjeldsen, H., Bedding, T. R., et al. 1998, A\&A, 335, 549

Zita, E. J. 1997, in ASP Conf. Ser. Vol. 135, A Half Century of Stellar Pulsation Interpretations, ed. P. A. Bradley \& J. A. Guzik (San Francisco: ASP), 414

Zita, E. J. 1999, in ASP Conf. Ser. Vol. 178, Stellar Dynamos: Nonlinearity and Chaotic Flows, ed. M. Nunez \& A. Ferriz-Mas (San Francisco: ASP), 203 